\title{
Impacto de una intervención educativa en el conocimiento sobre uso adecuado de antimicrobianos en infecciones respiratorias en un grupo de adolescentes
}

\author{
Beatriz Silva, Clarita Ferrada y M. Elena Santolaya
}

\section{Impact of an educational intervention on knowledge about appropriate use of antimicrobials in respiratory tract infections in adolescents}

Background: Knowledge about appropriate use of antimicrobials (AM) in the population is an important factor to achieve a proper use of these drugs. Objective: To evaluate the impact of an educational intervention on knowledge about appropriate use of AM in respiratory tract infections in adolescent from Lo Barnechea, Santiago. Methods: Prospective study. We evaluated the level of knowledge about appropriate use of AM in a group of adolescents from two municipal schools in Lo Barnechea. Then, in one of these schools, we made an educational intervention consisting of two workshops. Initial survey was repeated at the end of the first educational session and 4 months after completing the intervention. Results: We applied the knowledge survey to 489 students. The baseline rate of approval was $40.2 \%$. After the first workshop this percentage improved to $74.5 \%(\mathrm{P}=0.0001)$, and at 4 months after the educational program finished, the approval rate was 59.1\%. Conclusions: There are significant gaps in the level of knowledge about appropriate use of AM in the study population, which significantly improved after an educational intervention. Benefit was maintained for at least 4 months

Key words: Appropriate antibiotics use, knowledge, respiratory tract infections, educational intervention.

Palabras clave: Uso adecuado de antimicrobianos, infecciones respiratorias, conocimiento, intervención educativa.

\section{Introducción}

$\mathrm{E}$ xiste una alta tasa de uso de antimicrobianos en niños con infecciones respiratorias altas pese a que la mayoría de estas infecciones son de etiología viral, situación clínica en que no hay indicación para su uso $^{1-2}$. Se ha estimado que en 50 a $75 \%$ de las infecciones respiratorias virales de los niños los pacientes utilizan antimicrobianos, lo que constituye un uso inadecuado de estos medicamentos ${ }^{3,4}$.

La alta tasa de indicación de antimicrobianos en niños ha contribuido a incrementar la resistencia bacteriana en infecciones de la comunidad durante la última década, principalmente a penicilinas y macrólidos 5 . Se ha demostrado que la intervención de mayor eficiencia en prevenir el aumento de la resistencia es el uso racional de antimicrobianos y la vigilancia en su consumo ${ }^{3}$.

Existen muchos factores que influyen en el exceso de uso de antimicrobianos. Por parte del paciente y/o de sus padres existen expectativas pre-concebidas de recibir un antimicrobiano, y se conoce que la probabilidad de que un médico prescriba un antimicrobiano al atender a un niño es nueve veces mayor si éste cree que los padres desean dicha prescripción ${ }^{6}$. Se ha encontrado que al menos un tercio de las prescripciones de antimicrobianos por parte de pediatras en respuesta a la demanda de los padres son innecesarias ${ }^{7}$. Otras razones inapropiadas esgrimidas para su prescripción son evitar que la consulta se prolongue, una forma de terminar la consulta con pacientes disconformes, el temor a las consecuencias clínicas del no uso de antimicrobianos o la intención de prevenir infecciones secundarias $^{6,7}$.

Existen antecedentes en la literatura médica sobre la utilidad de las intervenciones educativas en la población en el nivel de conocimientos sobre uso adecuado de antimicrobianos. Al respecto, se ha visto que intervenciones educativas con estrategias únicas, como la difusión de material educativo (impresos, vía informática, charlas o métodos pasivos), no mejoran significativamente el nivel de conocimientos; en cambio se ha sugerido en algunas revisiones sistemáticas que estrategias que combinen charlas, talleres interactivos y material audiovisual, focalizados a los intereses y necesidades específicas del grupo intervenido, tienen mayor impacto en el nivel de conocimientos de la población intervenida, lo que podría a su vez contribuir a disminuir el uso de antimicrobianos ${ }^{9}$.

No encontramos evidencia sobre el grado de conocimientos de uso adecuado de antimicrobianos en
Hospital Luis Calvo Mackenna, Santiago, Chile. Servicio de Pediatría (BS). Universidad de Chile, Santiago. Facultad de Medicina Departamento de Pediatría, Campus Oriente (CF, MES).

Conflictos de interés: no hubo Financiamiento:

Recibido: 2 de noviembre de 2011 Aceptado: 29 de julio de 2012

Correspondencia a: María Elena Santolaya de Pablo msantola@med.uchile.cl 
población chilena ni sobre la utilidad de intervenciones educativas en niveles específicos de la población sobre este tema. El objetivo de este estudio fue evaluar el grado de conocimientos sobre uso adecuado de antimicrobianos en infecciones respiratorias en un grupo de adolescentes estudiantes de colegios públicos de la comuna de Lo Barnechea, y posteriormente, medir el impacto de una intervención educativa en el conocimiento sobre uso apropiado de antimicrobianos en este grupo.

\section{Materiales y Métodos}

\section{Tipo de estudio y población a estudiar}

Se realizó un estudio prospectivo e intervencional en un grupo de adolescentes de $7^{\circ}$ básico a $3^{\circ}$ año de enseñanza media de dos establecimientos educacionales municipalizados de la Comuna de Lo Barnechea, los colegios Complejo Educacional Eduardo Cuevas Valdés y Colegio Parroquial Santa Rosa. Los alumnos de estos colegios pertenecen al nivel socio-económico medio bajo, según definición de la Junta Nacional de Auxilio Escolar y Becas. Con respecto al rendimiento académico, ambos establecimientos obtuvieron un promedio de puntaje superior al promedio nacional según estrato socioeconómico en el SIMCE 2008. El estudio fue aprobado por el Comité de Ética científico pediátrico del Servicio de Salud Metropolitano Oriente y por los Directores de ambos establecimientos educacionales.

\section{Intervención}

En una etapa inicial se confeccionó un cuestionario de 10 preguntas de selección múltiple sobre conocimientos básicos en infecciones respiratorias, infecciones virales y bacterianas, nombres y posibles indicaciones de antimicrobianos y medidas de autocuidado ante infecciones respiratorias (Anexo 1). Este cuestionario se confeccionó con la ayuda de especialistas en educación en salud. Inicialmente se tomó un grupo de 30 estudiantes de niveles: $8^{\circ}$ básico y $1^{\circ}$ medio, con características socio económicas y culturales similares a las de la población a intervenir, a los que se les presentó el cuestionario para evaluar el grado de comprensión de lectura y de indicaciones generales. Según esto se modificó parcialmente la redacción de algunas preguntas. En una segunda etapa se aplicó el

Figura 1. Esquema de la intervención realizada y su evaluación. Octubre de 2009: test diagnóstico. Noviembre de 2009: Taller 1. Marzo de 2010: Taller 2. Julio de 2010: último test. cuestionario a los sujetos en estudio, de manera presencial, en su sala de clases, por dos de las investigadoras del estudio (BS, CF), acompañadas por el profesor jefe de cada curso. Se definió como aprobación un porcentaje de respuestas correctas $\geq$ a $60 \%$.

El cuestionario se aplicó a toda la población en estudio en un momento diagnóstico (octubre de 2009). Los resultados fueron presentados a los Directores de cada establecimiento educacional y se ofreció realizar estrategias educativas posteriores a esta primera evaluación. En uno de los dos colegios (Complejo Educacional Eduardo Cuevas Valdés), se nos permitió realizar estas estrategias educativas, consistentes en dos talleres, realizados en noviembre de 2009 y marzo de 2010. Cada sesión educativa consistió en un taller de $45 \mathrm{~min}$, en grupos de 20 a 30 estudiantes cada uno, realizados por las investigadoras, acompañadas con el profesor jefe. Cada taller se inició con una recapitulación de conocimientos previos poniendo énfasis en experiencias personales significativas; posteriormente se realizó trabajo de grupo (de 8 a 10 personas). Se utilizó un apoyo audiovisual para enfatizar los conceptos más importantes. Terminado el trabajo grupal se realizó una síntesis de lo aprendido y se dio un espacio para contestar preguntas. Finalizado cada taller se realizó una evaluación de los estudiantes al moderador del taller y viceversa. La encuesta diagnóstica inicial se volvió a aplicar en noviembre de 2009, inmediatamente después del primer taller y en julio de 2010, cuatro meses después de haber terminado la intervención (Figura 1).

\section{Análisis estadístico}

Para el análisis estadístico se utilizó el programa Stata 8.0. Se definió un error $\alpha$ de $5 \%$. Se calcularon los intervalos de confianza de los porcentajes de aprobación y promedios de puntajes para cada colegio y globales de toda la población a la que se le realizó la encuesta diagnóstica. Se realizó comparación de promedios y análisis de varianza para comparar puntajes según curso. En el análisis de las encuestas del colegio en que se realizó la intervención educativa se realizaron pruebas de independencia para análisis de datos categóricos. Para la comparación de porcentajes de aprobación antes y después en el grupo intervenido se realizó un análisis de muestras pareadas o repetidas.

\section{Resultados}

Se aplicó el cuestionario diagnóstico a 489 adolescentes de los dos colegios participantes. El porcentaje de aprobación fue de 40,2\%, siendo el puntaje promedio de 5,1 puntos de un máximo de 10 (DS: 1,75) con una mediana de 6 puntos. En el colegio en que se realizó la intervención educativa el cuestionario diagnóstico se aplicó a 219 niños con una tasa de aprobación de 39,7\% y un promedio de 5,1 puntos (DS: 1,8 ). 
Las preguntas con menor porcentaje de respuestas correctas fueron sobre uso adecuado de antimicrobianos; la pregunta con menor número de respuestas correctas $(15,1 \%)$ fue ¿En cuál de estas enfermedades el médico podría usar antibióticos?, seguida por ¿Para qué sirven los antibióticos? con 25,1\%. Por otra parte, las preguntas con mayor porcentaje de respuestas correctas fueron dos sobre autocuidado; la más acertada fue ¿Qué debo hacer si me duele el oído? con 79,9\% seguida de ¿Cómo puedo confirmar si una persona tiene fiebre? con $78,5 \%$ (Figura 2). Al analizar el porcentaje de aprobación según curso se vio que a mayor nivel académico mejora el resultado $(\mathrm{P}<0,01)$.

Con posterioridad al primer taller educativo se repitió el cuestionario inicial a los estudiantes intervenidos; la tasa de aprobación ascendió a $74,5 \%$ con un promedio de 6,9 puntos (DS: 2,09) y un aumento de aprobación de 34,7\% (P: 0,0001).

Se realizó por tercera vez el mismo cuestionario cuatro meses después de terminada la intervención educativa, obteniendo esta vez un porcentaje de aprobación de $59,1 \%$, con un promedio de 5,81 puntos (DS: 1,91 ) $\mathrm{y}$ un descenso de $15,4 \%$ respecto al test posterior al primer taller, diferencia estadísticamente significativa (p: 0,0001). Al comparar el porcentaje de aprobación diagnóstica versus la evaluación final, cuatro meses tras la intervención, se encontró un ascenso de 19,4\% (p: 0,0001) (Tabla 1, Figura 3).

\section{Discusión}

Existe una deficiencia importante de conocimientos básicos del uso adecuado de antimicrobianos en los adolescentes encuestados, predominantemente en los conceptos acerca de su utilidad. Esta deficiencia mejora significativamente después de realizar un taller educativo sobre el tema. Cabe destacar que este taller se basó en las experiencias personales de los alumnos e incorporó talleres grupales, material audiovisual y exposición resumida de los aspectos más importantes por parte del moderador.

Las preguntas con mayor porcentaje de respuestas correctas antes y después de la intervención fueron sobre auto-cuidado, lo que refuerza la importancia en el aprendizaje de las experiencias personales de cada alumno. Lo mismo se ve reflejado en el hecho de que a mayor escolaridad mejor porcentaje de aprobación a pesar de que estos contenidos no forman parte de programas del Ministerio de Educación.

El aumento en el grado de conocimientos se mantiene al menos cuatro meses después de finalizar la intervención, pero con un descenso significativo del porcentaje de aprobación del cuestionario. Esto sugiere la necesidad de un reforzamiento sistemático del aprendizaje en temas como el autocuidado y estilos de vida saludable en los

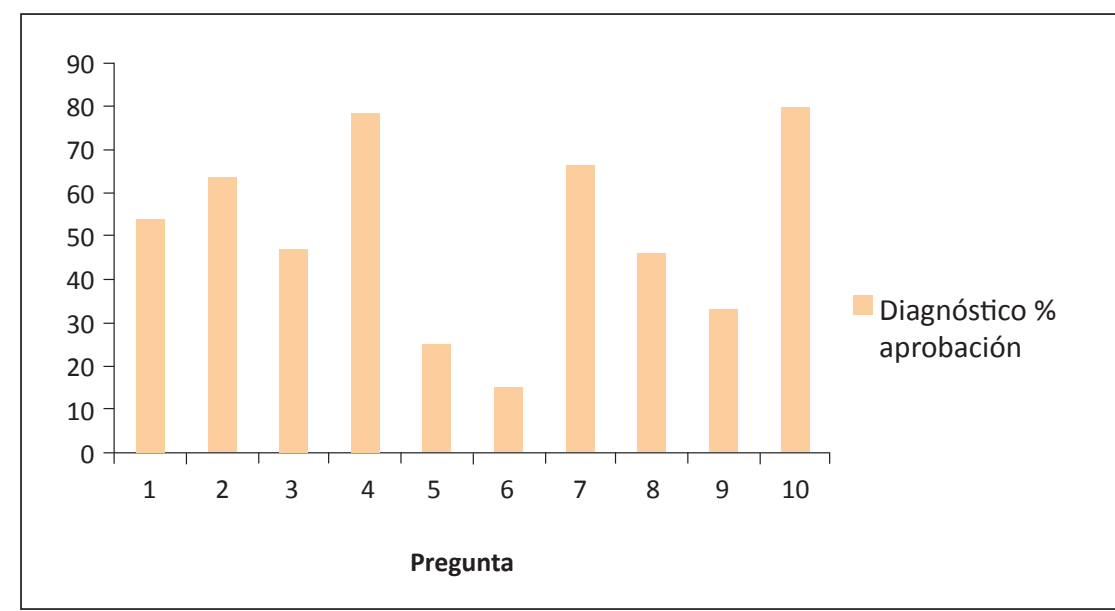

Figura 2. Porcentaje de aprobación por pregunta en la evaluación diagnóstica basal realizada a 489 estudiantes.

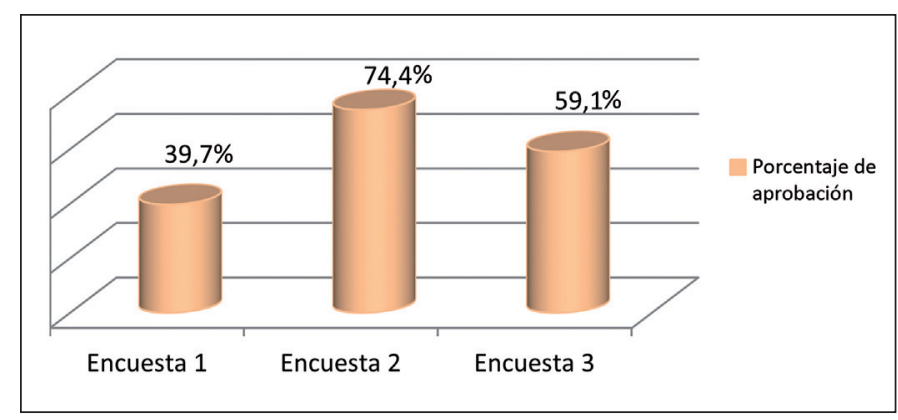

Figura 3. Porcentaje de aprobación del cuestionario basal, con posterioridad a primer y segundo taller educativo.

Tabla 1. Porcentaje de aprobación por pregunta para cada aplicación del cuestionario de conocimientos, preguntas especificadas en anexo 1

\begin{tabular}{cccc} 
Pregunta & $\begin{array}{c}\text { Basal } \\
\text { Aprobación (\%) }\end{array}$ & $\begin{array}{c}\text { Post } \mathbf{1}^{\circ} \text { taller educativo } \\
\text { Aprobación (\%) }\end{array}$ & $\begin{array}{c}\text { 4 meses después de } \mathbf{2}^{\circ} \\
\text { taller educativo } \\
\text { Aprobación (\%) }\end{array}$ \\
\hline 1 & 53,9 & 82,6 & 59,1 \\
\hline 2 & 63,5 & 84,2 & 74,4 \\
\hline 3 & 47,0 & 57,6 & 56,2 \\
\hline 4 & 78,5 & 94,0 & 87,7 \\
\hline 6 & 25,1 & 76,1 & 35,0 \\
\hline 7 & 15,1 & 38,0 & 20,2 \\
\hline 8 & 66,2 & 66,3 & 69,5 \\
\hline 9 & 46,1 & 67,9 & 52,2 \\
10 & 33,3 & 48,9 & 43,4 \\
\hline
\end{tabular}


programas educativos obligatorios del Ministerio de Educación.

Una debilidad de este estudio es que la población intervenida basalmente tiene mejores resultados en las evaluaciones SIMCE (Sistema de Medición de Calidad de la Educación) que otros colegios del mismo estrato socioeconómico de la comuna, lo que podría estar sobrevalorando la efectividad de la intervención.

La información obtenida ofrece una importante oportunidad de mejorar el grado de conocimientos en los adolescentes a través del trabajo integrado de los profesionales de educación y salud, lo que podría tener un impacto en el uso racional de antimicrobianos en la población.

\section{Resumen}

Antecedentes: El conocimiento sobre uso adecuado de antimicrobianos en la población es relevante para lograr un buen uso de estos medicamentos. Objetivo:
Evaluar el impacto de una intervención educativa en el conocimiento sobre uso adecuado de antimicrobianos en infecciones respiratorias en un grupo de adolescentes de la comuna de Lo Barnechea. Método: Estudio prospectivo. Se evaluó el grado de conocimientos sobre uso adecuado de antimicrobianos en estudiantes de $7^{\circ}$ básico a $3^{\circ}$ medio de dos colegios municipalizados de Lo Barnechea. Luego, en uno de estos establecimientos se realizó una intervención educativa consistente en dos talleres teórico-prácticos. Se repitió la encuesta inicial al terminar la primera sesión educativa y cuatro meses después de completar la intervención. Resultados: Se aplicó la encuesta de conocimientos a 489 estudiantes. El porcentaje basal de aprobación fue de 40,2\%. Luego del primer taller este porcentaje ascendió a 74,5\% (p: 0,0001); a los cuatro meses la tasa de aprobación fue de 59,1\%. Conclusiones: Existen carencias importantes en el grado de conocimientos sobre uso adecuado de antimicrobianos en la población estudiada, que mejora significativamente después de una intervención educativa, beneficio que se mantuvo al menos por 4 meses.

\section{Referencias bibliográficas}

1.- Marra F, Patrick D M, Chong M, Bowie W R. Antibiotic use among children in British Columbia, Canada. J Antimicrob Chemother 2006; 58: 830-9.

2.- McCaig L F, Besser R E, Hughes J M. Trends in antimicrobial prescribing rates for children and adolescents. JAMA 2002; 287: 3096-102.

3.- Bavestrello L, Casanova D. Impacto de medidas regulatorias en la tendencia de consumo comunitario de antibióticos en Chile. Rev Med Chile 2002; 130: 1265-72.

4.- Mohamad G, Raymond C, Ruth T, Louis D. Do resident physicians use antibiotics appropriately in treating upper respiratory infections? A survey of 11 Programs. Clin Infect Dis 2003; 37: 853-6.

5.- Antibiotic resistance and prescribing practice. National Prescribing Centre NHS, MeRec Briefing Issue $\mathrm{N}^{\circ} 21$. http://www.npc.nhs.uk/ merec/infect/commc

6 .- Huang S, Rifas-Shiman S, Kleinman K,
Kotch J, Schiff N. Parental knowledge bout antibiotic use: results of a clusterrandomized, multicommunity intervention. Pediatrics 2007; 119: 698-706.

7.- Finkelstein J, Huang S, Kleimann K, Rifas-Shiman S, Stille C, Daniel J, et al. Impact of a 16- community trial to promote judicious antibiotic use in Massachusetts. Pediatrics 2008; 121; e15-e23.

8.- Arnold S R, Straus S E. Interventions to improve antibiotic prescribing practices in ambulatory care (Cochrane Review). In: The Cochrane Library, Issue 2, 2008. 
1. ¿Cuál es la principal causa del resfrío común?
A. Virus.
B. Bacterias.
C. Enfriarse.
D. Tener el pelo mojado por mucho tiempo.

2. ¿Hasta cuánto tiempo puede durar como máximo un resfrío?
A. 2 días.
B. 10 días.
C. 20 días.
D. 30 días.

3. ¿Qué debo hacer si estoy resfriado?
A. Usar un antibiótico.
B. Usar aspirina.
C. Usar vitamina C.
D. Tomar líquidos abundantes y ningún medicamento.

4. ¿Cómo puedo confirmar si una persona tiene fiebre?
A. Poniendo la mano en su frente para ver si está más caliente que lo normal.
B. Cuando la persona se queja de molestias como sudoración o dolor de cabeza.
C. Tomando la temperatura con termómetro en su axila.
D. Poniendo los labios en su frente para ver si está más caliente que lo normal.

5. ¿Para qué sirven los antibióticos?
A. Para las infecciones producidas por bacterias.
B. Para las infecciones producidas por virus.
C. Para la fiebre.
D. Para prevenir el contagio del resfrío

6. ¿En cuál de estas enfermedades el médico podría usar antibióticos?
A. Resfrío.
B. Bronquitis viral con flemas verdes
C. Sinusitis aguda.
D. Faringo-amigdalitis viral

7. ¿Qué puedo hacer para evitar que aumente la resistencia de las bacterias a los antibióticos?
A. Pedir al médico antibióticos cuando asista a su consulta.
B. Si me recetan un antibiótico tomarlo exactamente los días que me indicó el médico.
C. Dejar de tomar el antibiótico cuando me sienta mejor.
D. Guardar lo que me sobre de antibiótico para la próxima vez que esté enfermo.

8. ¿Cuál de los siguientes medicamentos es un antibiótico?
A. Amoxicilina.
B. Paracetamol.
C. Ibuprofeno.
D. Nastizol.

9. La inyección de penicilina benzatina por una vez sirve para:
A. Resfrío.
B. Sinusitis.
C. Fiebre.
D. Faringo-amigdalitis bacteriana.

10. ¿Qué debo hacer si me duele el oído?
A. Usar tapones de algodón.
B. Usar por mi cuenta antibióticos.
C. Usar cucuruchos con humo de cigarro.
D. Consultar a un médico. 